\title{
Cherenkov-channeling radiation by relativistic muons in crystals
}

\author{
K. B. Korotchenko ${ }^{1}$, E. I. Rozhkova ${ }^{1}$, S. B. Dabagov ${ }^{2,3,4, a}$ (i) \\ ${ }^{1}$ NR Tomsk Polytechnic University, Lenin Ave. 30, 634050 Tomsk, Russia \\ 2 INFN-Laboratori Nazionali di Frascati, Via E. Fermi 40, 00044 Frascati, RM, Italy \\ ${ }^{3}$ NR Nuclear University MEPhI, Kashirskoe Sh. 31, 115409 Moscow, Russia \\ ${ }^{4}$ RAS-P.N. Lebedev Physical Institute, Leninsky Pr. 53, 119991 Moscow, Russia
}

Received: 27 May 2020 / Accepted: 21 September 2020 / Published online: 8 October 2020

(C) The Author(s) 2020

\begin{abstract}
In this work we analyse Cherenkov radiation by relativistic muons, positive and negative, channeled in optically transparent diamond and silicon crystals in comparison with ordinary Cherenkov radiation. We have shown that the maxima in the spectral angular distributions for both types of radiation are revealed at the derivative extrema for the media refractive index, while, due to the difference in scattering of positively and negatively charged particles at crystal channeling, the number of Cherenkov photons emitted by channeled positive muons might be over the one for negative muons. We have demonstrated that Cherenkov radiation by quasi free projectiles is described as one limiting approximation of a general expression for Cherenkov radiation by channeled projectiles, which takes into account non-zero derivative of the refractive index. The last may result in essential increase of radiation intensity.
\end{abstract}

\section{Introduction}

Near future research programs in physics are strongly related to new developments in muon physics and thus mainly defined by advances in muon beams accelerator, storage and beam shaping techniques [1]. Any new peculiarities to be registered at relativistic muon interaction in a media may become a powerful instrument for various applications. However, even well-known phenomena applied at new conditions might be of growing interest for planning future experiments.

In our recent work [2] in terms of quantum electrodynamics we have modified well-known expression for Cherenkov radiation $(\mathrm{ChR})$, which differs from the standard one by a factor that takes into account the media dispersion (see e.g. [3-5]). It was shown that a newly derived formula represents one of the limiting approximations of more general expression, while other approach in calculations leads us to another known radiation, so-called channeling radiation (CR). In those both cases the large emission angles have been considered. We have shown that the electromagnetic radiation at large angles by relativistic channeled particles appears to be a new type of optical radiation - Cherenkov-channeling radiation (ChCR).

Cherenkov radiation is one of the recognised phenomena utilised for muons registration (e.g. so-called Cherenkov detectors) [6]. Hence, hopefully ChCR could be also efficient for detecting muons, and muons states as well. The last is rather important in studying various processes, for instance, in the process of muon-to-electron transition, which takes place in a muon atom [7].

On the other hand, spontaneous CR in optical region becomes feasible in crystals with the refractive index $n>1$ (see [8]). It can be observed at large angles to the longitudinal momentum of a channeled particle, close to Cherenkov angle for a given projectile [9-12]. Due to the mass-factor, CR for heavy projectiles typically does not represent notable interest as a radiation source [13], while CR at large angles might be very useful for a beam diagnostics. For instance, it would be interesting to apply theoretical discoveries of this work to muon channeling, which recently was successfully realised at Fermilab muon collider [14,15].

Previously we have shown that the intensity of ChCR by light particles, namely, by electrons, might be much higher than ChR [2]. In this report, in view of the importance for developing novel radiation-based tools, we present our results in studying both ChCR and CR by much heavier particles, i.e. by muons. We also analyse the properties of ultraviolet ChCR by relativistic muons in optically transparent crystals that takes into account the crystal dispersion $n=n(\omega)$.

\footnotetext{
a e-mail: sultan.dabagov@lnf.infn.it (corresponding author)
} 


\section{ChR by muons for non-zero media dispersion}

As recently shown [2], channeled relativistic particles emit $\mathrm{ChCR}$ at the angles close to Cherenkov ones. The expression for standard ChR derived from a general definition of ChCR after the summation over all quantum states of the channeling motion in aligned crystal

$d N_{C h R} \simeq \frac{\alpha}{c \hbar} \beta\left(1-\frac{1}{n^{2} \beta^{2}}\right) G(\omega)$

under the condition $\cos \theta_{C h}=1 /(n \beta)$ can be simplified to the following expression

$d N_{C h R} \simeq \frac{\alpha}{c \hbar} \beta \sin ^{2} \theta_{C h} G(\omega)$,

where $\alpha$ is the fine structure constant, $c$ is the speed of light in a vacuum, $\hbar$ is the reduced Planck constant, $\beta=v / c$ is the normalised particle velocity, $\theta_{C h}$ is the Cherenkov angle, and $G(\omega)=1+(\omega / n(\omega))(\partial n / \partial \omega)$ is the function, which takes into account non-zero dispersion function for a given media.

Equations (1) and (2) at $G(\omega)=1$ exactly match the ChR angular distribution reduced within standard ChR theory (see e.g. $[3-5,16,17])$. To analyse these equations as functions of the factor $G(\omega)$, we consider two optically transparent crystals:

- a C (diamond) crystal as one of the most unique optically transparent crystal for the frequency range from $\sim 220 \mathrm{~nm}(\sim 5.6 \mathrm{eV})$ up to microwaves [18];

- a Si crystal with one of the largest value of the refractive index $n(\omega)$ and its derivative $\partial n(\omega) / \partial \omega$ as well [18]. Ultra thin Si crystal (thickness $L<1 \mu \mathrm{m}$ ) is optically transparent.

Figure 1 shows measured dependencies for the real part of refractive index versus photon frequency (energy) for chosen $\mathrm{C}$ and Si crystals [18] as well as the dependences of Cherenkov angle $\theta_{C h}=\arccos [1 /(n \beta)]$ as function of photon energy. The use of these data in combination with Eqs. (1) and (2) allows the number of ChR photons emitted per unit path of relativistic particle to be easily calculated for a whole Cherenkov cone.

The results of calculations for $10 \mathrm{GeV}$ muons are presented in Fig. 2. To compare, the dashed curve indicates similar results obtained by the standard formula at $G(\omega)=1$, i.e. without taking into account the crystal dispersion. In a Si crystal the maximum number of ChR photons, $d N_{C h R}^{\max } \simeq$ $1740 \mathrm{ph} /(\mathrm{eV} \mathrm{cm})$ at $\hbar \omega \simeq 3.2 \mathrm{eV}$ is about 5 times greater compared to the number of ChR photons calculated using the standard expression, while in a $\mathrm{C}$ crystal the maximum, $d N_{C h R}^{\max } \simeq 1010 \mathrm{ph} /(\mathrm{eV} \mathrm{cm})$, observed at $\hbar \omega \simeq 7 \mathrm{eV}$ shows near triple increase.

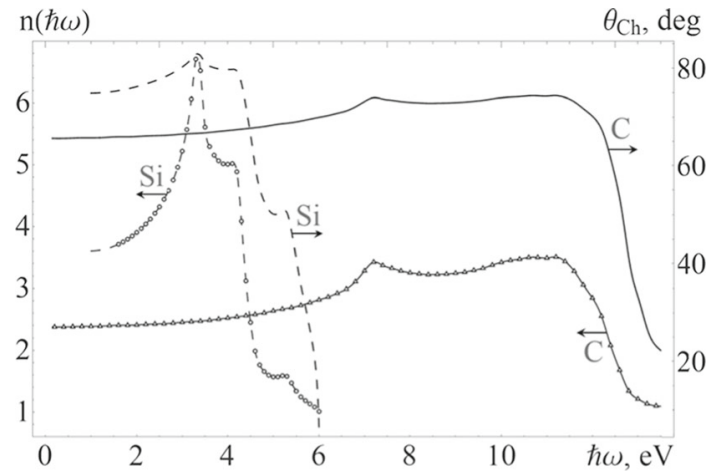

Fig. 1 Refractive indexes $n=n(\omega)$ (dot-solid lines related to the left ordinate) and Cherenkov angles versus ChR photons energy (solid and dashed lines related to the right ordinate) for both $\mathrm{C}$ and $\mathrm{Si}$ crystals. Dots indicate the experimental data [18]

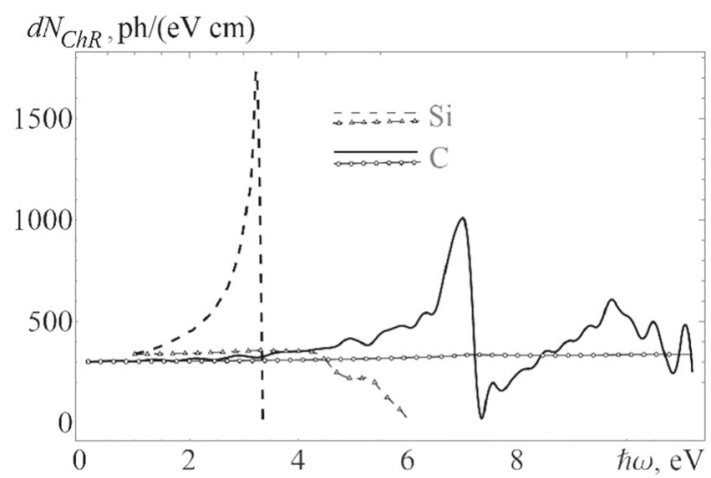

Fig. 2 Spectral distributions of $\mathrm{ChR}$ photons per unit path and unit energy calculated for both C (diamond) and Si crystals with the dispersion taken into account (solid line) and without it (at $G(\omega)=1$, dashed line)

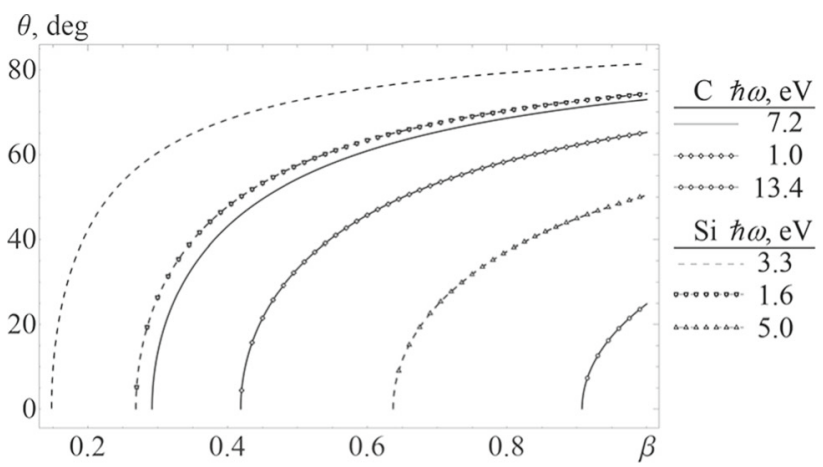

Fig. 3 Dependency of Cherenkov angles $\theta_{C h}$ on particle velocity $\beta=$ $v / c$ for both $\mathrm{C}$ (diamond) and $\mathrm{Si}$ crystals

As mentioned in [19], the fact that Cherenkov angle $\theta_{C h}$ is strictly dependent on the particle velocity $\beta$, is of fundamental importance for various applications [20-23]. This reliance sets the design constrains on experimental setups for $\mathrm{ChR}$ registration. Figure 3 demonstrates the function $\theta_{C h}$ versus $\beta$ for non-zero crystal dispersion. 
These dependences confirm a well-known feature of Cherenkov angle $\theta_{C h}$ in a media with $n(\omega)$ to tend to the limit value [19], becoming independent of the particle velocity $\beta$. Taking into account the total internal reflection at the air-crystal interface, we can conclude that the discussed behaviour of ChR in crystals is not suitable for muon detectors in a wide range of energies (e.g. ring-imaging Cherenkov detectors). This finding is valid also for other relativistic particles.

However, the use of optically transparent crystals with high refraction indexes may drastically change situation providing various applications with reasonable $\mathrm{ChR}$ intensities.

\section{Spontaneous CR by muons}

The radiation intensity by various projectiles in crystals may reveal an essential increase for different spectral and angular intervals at specific channeling conditions $[8,10]$. Typically this postulate is true for radiation emitted under small angles to the projectile momentum ("forward radiation"). However, bound channeling motion can contribute effectively to other known radiation phenomena observed at large angles [2]. This fact, mostly proved for light particles, should be also valid for relativistic muons independently of notable mass factor. Below in brief we remind properties of a new type of radiation, mixed $\mathrm{ChCR}$, but applied for the case of muon planar channeling.

The number of ChCR photons within the solid angle $\Delta o$ emitted by a channeled muon $\mu$ per unit length and unit photon energy during its transition $i \rightarrow f$ between the quantum states of transverse motion (at $i \neq f$ ) can be estimated by the following expression $[11,12]$

$$
\begin{aligned}
d N & =\sum_{i, f} \frac{1}{c \hbar} \frac{1}{\hbar \omega} \frac{d I_{i f}}{d \omega \Delta o}, \\
\frac{d I_{i f}}{d \omega \Delta o}= & \frac{e^{2} x_{i f}^{2} \Omega_{i f}^{2} \omega}{4 c^{3} \pi \beta^{3} n^{2}(\omega)} P_{i}\left(\theta_{0}\right) G(\omega) \Theta\left(W_{\Delta}\right) \Theta\left(W_{0}\right) \\
& \times\left[\Delta^{+}\left(1-2 \frac{\omega}{\omega_{m}}+\left(\frac{\omega}{\omega_{m}}\right)^{2}\right)+n^{2}(\omega) \beta^{2} \Delta^{-}\right],
\end{aligned}
$$

where $e$ is the electron charge (the charge of a muon $\mu$ ), $x_{i f}=\left\langle f\left|x e^{-\mathbf{i} \kappa_{x} x}\right| i\right\rangle$ is the matrix element for the muon $\mu$ transition $i \rightarrow f$ with the $x$-projection of the emitted photon wave vector $k_{x}, \hbar \Omega_{i f}=\varepsilon_{i}-\varepsilon_{f}$ is the photon energy in a rest system, $\omega_{m}$ is the maximum observed radiation frequency.

Such simplification (at $i \neq f$ ) is suitable when ChCR photon energy is essentially less than the energy of channeled relativistic muon [2]. In this approximation the matrix elements $x_{i f}$ can be calculated in a dipole approximation, while the number of ChCR photons for intrazone transitions $(i=f)$ has to be calculated utilising the expression of Eq. (1).

The solid angle $\Delta o$ corresponds to the photon detector elementary area, $P_{i}\left(\theta_{0}\right)$ denotes to the initial population of the $i$-th transverse quantum state as a function of the incidence angle $\theta_{0}=\arctan \left[p_{x} / p_{z}\right]$ with respect to the channeling planes. Other notations in Eq. (5) are as follows

$\Delta^{ \pm}=(\Delta \varphi \pm \cos (\Delta \varphi+2 \varphi) \sin \Delta \varphi)$,

and the arguments of the Heaviside's function $\Theta(\ldots)$ -

$W_{\Delta}=\frac{\Omega_{i f}}{1-n(\omega) \beta \cos (\theta+\Delta \theta)}-\omega$,

$W_{0}=\omega-\frac{\Omega_{i f}}{1-n(\omega) \beta \cos \theta}$

are determined by the detuning from the resonance frequencies.

\section{ChCR by planar channeled $\mathrm{GeV}$ muons}

The analysis of ChCR is performed for $10 \mathrm{GeV}$ muons, $\mu^{-}$ and $\mu^{+}$, channeled along (110) planes in both Si and C (diamond) crystals. All calculations for the angular and spectral distributions of the ChCR photons number $d N$ are carried out using the Eqs. (1), (3) and (5) for the photon beam collimator $\Delta \theta=\Delta \varphi=0.3 \mathrm{mrad}$ that is chosen as typical angular size of the area unit cell for the photon detector.

The angular distributions of ChCR-photons number $d N$ at fixed energies, i.e. $\hbar \omega \sim 3.3 \mathrm{eV}$ in Si with $\theta_{C h}^{\max } \simeq 81.4^{\circ}$ and $\hbar \omega \simeq 7.2 \mathrm{eV}$ in $\mathrm{C}$ (diamond) with $\theta_{C h}^{\max } \simeq 73.0^{\circ}$ that correspond to the extrema in ChR photons spectra (Fig. 2), are shown in Fig. 4. In both cases we can reveal the influence of channeling on the radiation spectra. ChCR by planarchanneled $\mu^{+}$is more intense than those by $\mu^{-}$, and, moreover, in the spectra for $\mu^{+}$we can clearly resolve a few side peaks. These results confirm the admitted difference for channeling of positively and negatively charged particles, namely, a positively charged projectile at planar channeling displays longer life time in various quantum states.

Table 1 collects values of the polar angles $\theta$ that correspond to the maximum number $d N^{\max }$ of ChCR-photons that come to the photon detector within the angular cone $\Delta \theta=\Delta \varphi=0.3 \mathrm{mrad}$. These data prove general conclusion that the angles for maxima ChCR are slightly over those for ordinary ChR under other similar conditions.

Further calculations are devoted to the spectral distributions of the ChCR photon number for the polar angles $\theta$ given in Table 1. The results are shown in Fig. 5. From the plots we can see that the ChCR spectral distribution simulated for some fixed angle forms by contribution of various photons with different energies appearing in known angular distributions (Fig. 4). This peculiarity is most pronounced for both 

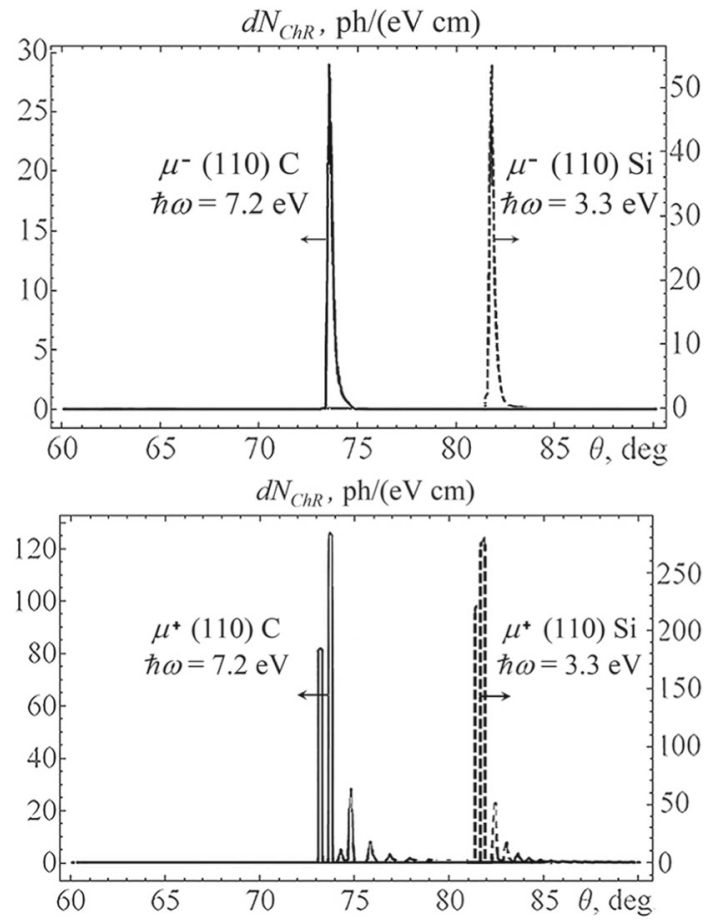

Fig. $4 \mathrm{ChCR}$ angular distributions at the energies corresponding to the maximum values of $G(\omega)$ (see Fig. 2). ChCR photons are emitted by channeled $10 \mathrm{GeV}$ muons $\left(\mu^{-}\right.$and $\left.\mu^{+}\right)$: (right) $\hbar \omega \sim 3.3 \mathrm{eV}$ in $\mathrm{Si}$ $\left(\theta_{C h}^{\max } \simeq 81.4^{\circ}\right) ;($ left $) \hbar \omega \simeq 7.2 \mathrm{eV}$ in diamond $(\mathrm{C})\left(\theta_{C h}^{\max } \simeq 73.0^{\circ}\right)$

types of muons $\left(\mu^{+}\right.$and $\left.\mu^{-}\right)$in a $\mathrm{C}$ crystal. The sharp maxima are observed near the radiation energy $\hbar \omega \sim 10 \mathrm{eV}$.

This analysis on ChCR spectral-angular distributions manifests once more that the maxima of ChCR by their photon both energies and numbers are determined by the $G(\omega)$ extrema.

Another interesting feature of $\mathrm{ChCR}$ relates to the fact that the channeling mode allows expanding the range of angles, at which this type of radiation is allowed [2]. To study similar observation for muons, we have calculated the angular distributions of ChCR for fixed photon energies 7.2 and $5.6 \mathrm{eV}$ emitted by positively charged muons $\mu^{+}$channeled in a $\mathrm{C}$ crystal. Figure 6 presents those distributions for the angles greater than $\theta>73^{\circ}$.

Table 1 Maximum number of ChCR photons (hitting the photon detector) emitted by $10 \mathrm{GeV}$ muons planar channeled in both $\mathrm{C}$ (110) and Si (110) crystals and related polar angles

\begin{tabular}{llllc}
\hline & $\hbar \omega(\mathrm{eV})$ & $\mu$ & $\theta(\mathrm{deg})$ & $d N_{C h C R}^{\max }[\mathrm{ph} /(\mathrm{eV} \mathrm{cm})]$ \\
\hline $\mathrm{C}$ & 7.2 & $\mu^{-}$ & 73.45 & 28 \\
& & $\mu^{+}$ & 73.64 & 126 \\
$\mathrm{Si}$ & 3.3 & $\mu^{-}$ & 81.77 & 54 \\
& & $\mu^{+}$ & 81.82 & 282 \\
\hline
\end{tabular}
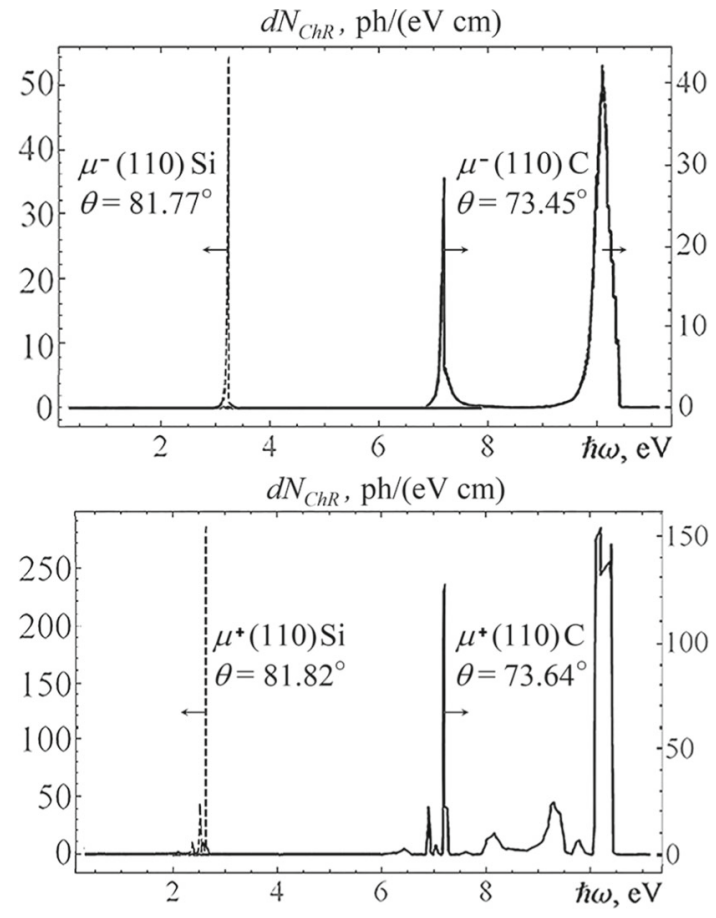

Fig. 5 Spectral distribution of ChCR by channeled $10 \mathrm{GeV}$ muons $\left(\mu^{-}\right.$and $\mu^{+}$) at the polar angle $\theta$ (from Table 1): $\theta \simeq 81.77^{\circ}\left(\mu^{-}\right)$and $\theta \simeq 81.82^{\circ}\left(\mu^{+}\right)$in $\mathrm{Si} ; \theta \simeq 73.45^{\circ}\left(\mu^{-}\right)$and $\theta \simeq 73.64^{\circ}\left(\mu^{+}\right)$in $\mathrm{C}$ (diamond)
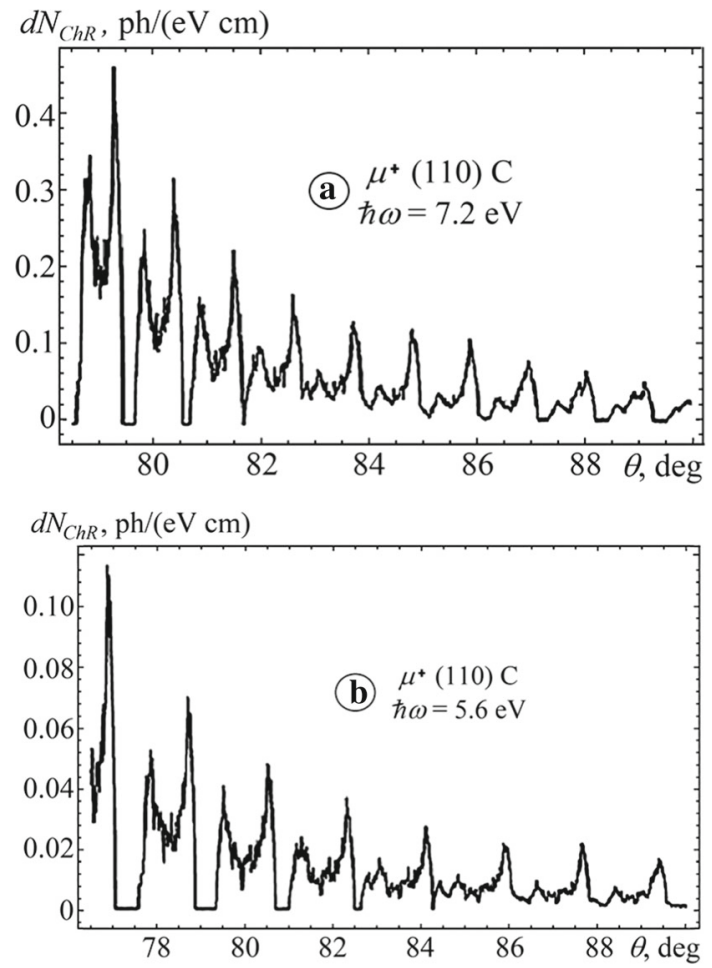

Fig. 6 ChCR angular distributions for two chosen energies $7.2 \mathrm{eV}$ and $5.6 \mathrm{eV}$ emitted by $10 \mathrm{GeV} \mu^{+}$channeled in $\mathrm{C}$ crystal (for angles greater than $\theta>73^{\circ}$ ) 
Table 2 Maximum number of ChCR photons hitting the photon detector of the angular size $\Delta \theta=\Delta \varphi=0.3 \mathrm{mrad}$ emitted by $\mu^{-}$and $\mu^{+}$ muons in both $\mathrm{C}$ and $\mathrm{Si}$ crystals under the azimuth angle $\theta$

\begin{tabular}{llllc}
\hline & $\hbar \omega(\mathrm{eV})$ & $\mu$ & $\theta(\mathrm{deg})$ & $d N_{C h C R}^{\max }[\mathrm{ph} /(\mathrm{eV} \mathrm{cm})]$ \\
\hline $\mathrm{C}$ & 5.60 & $\mu^{-}$ & 69.13 & 8.07 \\
& & $\mu^{+}$ & 69.48 & 23.34 \\
$\mathrm{Si}$ & \multirow{2}{*}{2.07} & $\mu^{-}$ & 75.96 & 1.20 \\
& & $\mu^{+}$ & 76.33 & 11.26 \\
\hline
\end{tabular}

We would like to underline here that the number of ChCR photons $d N$ for these energies almost coincides with the number $d N_{C h R}$ of Cherenkov photons for the same energies (see below in Table 3). However, ChR photons by quasi free projectiles are not emitted under such large angles. The maximum value of Cherenkov angle for ChR photons emitted by relativistic $10 \mathrm{GeV}$ particles in $\mathrm{C}$ crystals equals to $\theta_{C h}^{\max } \simeq 73.46^{\circ}$ and corresponds to the photon energy $\hbar \omega \simeq 11.3 \mathrm{eV}$.

As known, a C crystal is optically transparent for photon energies $\hbar \omega \leq 5.6 \mathrm{eV}(220 \mathrm{~nm})$, while for a ultrathin Si crystal of $L<1 \mu \mathrm{m}$ thickness we get $T>75 \%$ transparency for photon energies $\hbar \omega \leq 2.1 \mathrm{eV}(600 \mathrm{~nm})$ [18]. Due to such high transmission characteristics we have plotted the angular distributions of ChCR in those crystals at fixed photon energies (Fig. 7). The maximum numbers $d N^{\max }$ of ChCRphotons hitting the photon detector with solid angle $\Delta o$ and corresponding polar angles are shown in Table 2, separately for C and Si crystals as well as for $\mu^{+}$and $\mu^{-}$.

These data demonstrate again higher emission ability of $\mu^{+}$with respect to $\mu^{-}$that takes place due to the essential difference in the projectile phase redistribution for positively and negatively charged particles in the transverse plane of channeling. The dechanneling length for $\mu^{+}$at planar channeling is over then the length for $\mu^{-}$that makes the spontaneous radiation by the first one intenser $[10,13]$.

Successfully we have simulated the ChCR spectral distributions for fixed polar angles $\theta$, at which the angular spectra show the maxima (see Table 2). These spectra are presented in Fig. 8. According to these results at polar angles $\theta$ corresponding to the threshold of the crystal optical transparency, the ChCR spectral distributions by muons channeled in both $\mathrm{C}$ and Si crystals are qualitatively similar. However, ChCR by negative muons $\mu^{-}$reveals a wide range of continuity into the visible range. Indeed, the $\mathrm{ChCR}$ spectral distribution at $75.96^{\circ}$ by negative muons $\mu^{-}$channeled in (110) $\mathrm{Si}$ (see Fig. 8a) shows the widest range of continuity into the visible range of spectrum. It may be the main practical advantage of ChCR compared to ChR. It is important to emphasize that the number of ChCR photons for this spectrum is not less than the number of $\mathrm{ChR}$ photons.
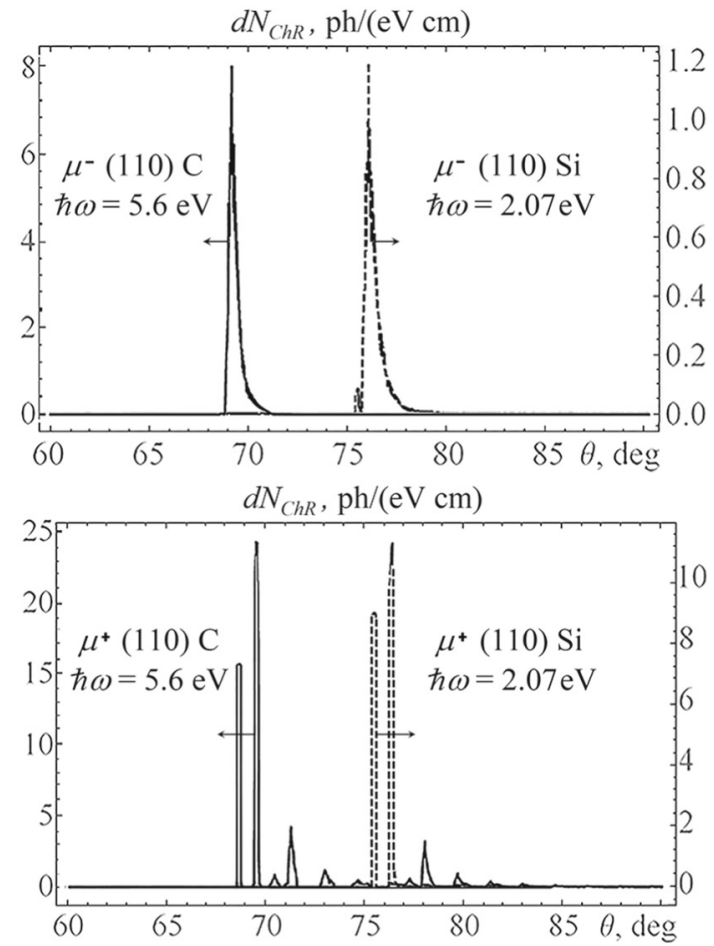

Fig. 7 ChCR angular distributions for the frequencies of optical crystal transparency $2.07 \mathrm{eV}$ for $\mathrm{Si}$ and $5.6 \mathrm{eV}$ for $\mathrm{C}$
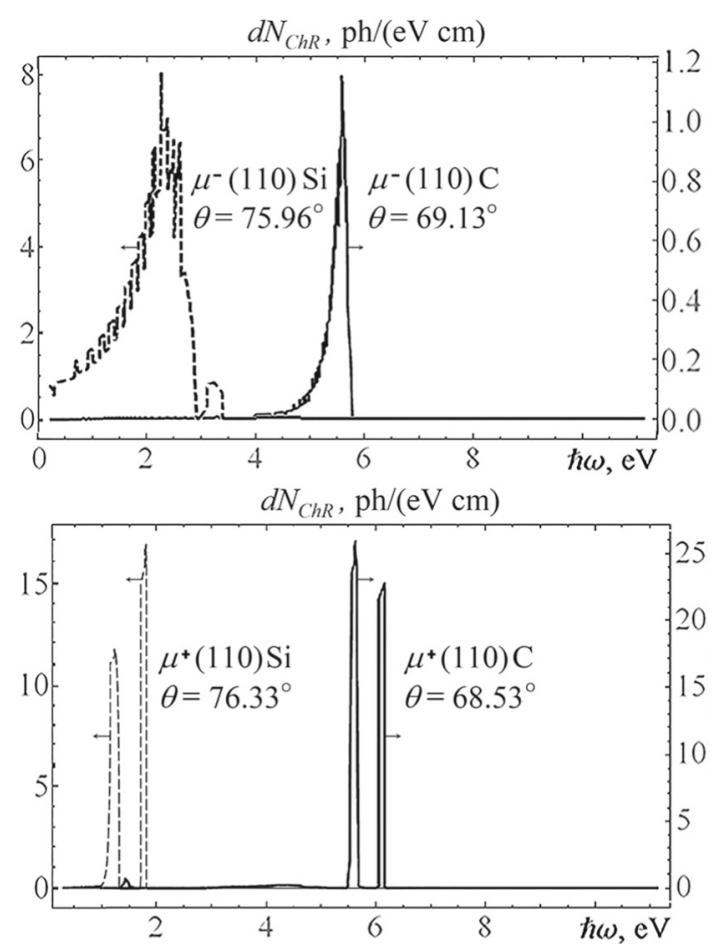

Fig. 8 Spectral distributions of ChCR photons emitted at polar angles $\theta$ corresponding to the threshold of crystals optical transparency (see Table 2) 
Table 3 Maximum number of ChR-photons hitting the photon detector of the angular size $\Delta \theta=\Delta \varphi=0.3 \mathrm{mrad}$ emitted by $\mu^{-}$and $\mu^{+}$muons in both $\mathrm{C}$ and $\mathrm{Si}$ crystals at the angles $\theta_{\mathrm{Ch}}^{\max }$

\begin{tabular}{llll}
\hline & $\hbar \omega(\mathrm{eV})$ & $\theta_{C h}^{\max }(\mathrm{deg})$ & $d N_{C h R}^{\max }[\mathrm{ph} /(\mathrm{eV} \mathrm{cm})]$ \\
\hline $\mathrm{C}$ & 7.20 & 73.0 & 0.162 \\
& 5.60 & 68.5 & 0.153 \\
$\mathrm{Si}$ & 3.30 & 81.4 & 0.173 \\
& 2.07 & 75.3 & 0.165 \\
\hline
\end{tabular}

To compare results for ChCR and ChR photons, in Table 3 the maximum numbers $d N_{C h R}^{\max }$ and Cherenkov angles $\theta_{C h}$ of ChR-photons versus the energy $\hbar \omega$ are presented. Reported data prove that the maximum numbers $d N_{C h R}^{\max }$ of ordinary ChR photons emitted by quasi free negative $\mu^{-}$and positive $\mu^{+}$muons crossing $\mathrm{Si}$ and $\mathrm{C}$ crystals are much smaller than the numbers $d N^{\max }$ of energy equivalent ChCR photons emitted by channeled muons (see Tables 1 and 2).

\section{Conclusions}

In this work we have presented our first results of theoretical studies on Cherenkov radiation by relativistic muons at channeling in optically transparent monocrystals, comparison of that with ordinary Cherenkov radiation under other equivalent conditions makes evident the advantages of newly proposed radiation.

We have shown for the first time that the energies (as well as the polar angles of radiation) corresponding to the largest number of photons for $\mathrm{ChR}$, and $\mathrm{ChCR}$ as well, emitted by muons are determined by extrema of the function $G(\omega)=$ $1+\frac{\omega}{n(\omega)} \frac{\partial n}{\partial \omega}$, which depends on the media dispersion.

Obtained results have demonstrated that the channeling process may significantly extend the angular fan for ultraviolet frequencies of photon emission by relativistic muons.

We have confirmed the fact that positively charged projectiles at channeling should radiate more intensively than negative ones, valid also for large emission angles. Indeed, due to longer life time in a channeling regime for positive $\mu^{+}$ muons with respect to negative $\mu^{-}$ones, i.e. $\mu^{+}$are characterised by larger dechanneling length compared $\mu^{-}$and emit more ChCR photons. This is the result of a strong phase redistribution of the beam in transverse plane of channeling motion at Coulomb scattering in the periodic field of averaged continuous potentials formed by crystal planes or axes (in this work we have examined only planar channeling case).

In our studies we have discovered that some tail of the ChCR spectrum by relativistic muons may end into the visible frequencies. ChCR by $10 \mathrm{GeV}$ negative $\mu^{-}$muons planar channeled in $\mathrm{Si}(110)$ reveals the widest range of continuity in the visible spectrum.
And finally, we have to note another interesting phenomenon, out of the scope of this work, related to the visible spectrum of ChCR emitted by relativistic muons channeled in $\mathrm{C}$ crystals, studies on which will be the subject for a separate paper.

Acknowledgements We express our gratitude to the reviewers and the editor as well for useful comments on improving our manuscript. This research is carried out by the TPU, MEPhI and LNF collaboration within the framework of the TPU Competitiveness Enhancement Program grant. One of the authors (SBD) would like to acknowledge the support by the Competitiveness Program of the NR Nuclear University MEPhI (Moscow). When the revised version of this manuscript was ready for submission, one of the authors of the concept for Cherenkovchanneling radiation, our dear colleague and close friend, Yury Pivovarov suddenly passed away. We dedicate this work to his memory.

Data Availability Statement This manuscript has no associated data or the data will not be deposited. [Authors' comment: There is no data online available because our work is mostly analytical. For any detail please contact the corresponding author.]

Open Access This article is licensed under a Creative Commons Attribution 4.0 International License, which permits use, sharing, adaptation, distribution and reproduction in any medium or format, as long as you give appropriate credit to the original author(s) and the source, provide a link to the Creative Commons licence, and indicate if changes were made. The images or other third party material in this article are included in the article's Creative Commons licence, unless indicated otherwise in a credit line to the material. If material is not included in the article's Creative Commons licence and your intended use is not permitted by statutory regulation or exceeds the permitted use, you will need to obtain permission directly from the copyright holder. To view a copy of this licence, visit http://creativecomm ons.org/licenses/by/4.0/. Funded by $\mathrm{SCOAP}^{3}$.

\section{Appendix A: Discussion on optical Cherenkov radiation by muons in crystals}

Because of high indexes of refraction at optical frequencies for a $\mathrm{C}$ (diamond) (or Si) crystal (Fig. 1), in general scheme of a flat crystal and a normal muon incident angle, ChCR would be totally reflected at the output crystal surface resulting in no radiation emission from the crystal into the vacuum. This problem can be resolved if the crystal is prepared in an appropriate manner to be successfully aligned at dedicated channeling direction.

If the crystal is cut in a way that the planes for channeling, for instance (110), are directed at angle $\alpha_{0}$ with respect to the crystal surface (Fig. 9), then the emission (exit) angle from the crystal $\beta$, as known, is defined by the equation

$n(\omega) \cos \theta=\sin \beta$,

where $\theta$ is the angle of emission for a ChCR photon of the energy $\hbar \omega$.

As an example, Fig. 10 demonstrates the dependences of the emission (exit) angle $\beta$ on the muons inlet angle $\alpha_{0}$ for 


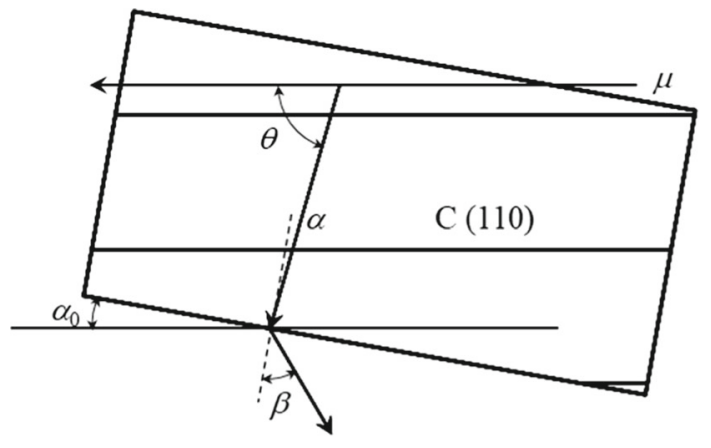

Fig. 9 One possible scheme of muon channeling in a specially fabricated crystal

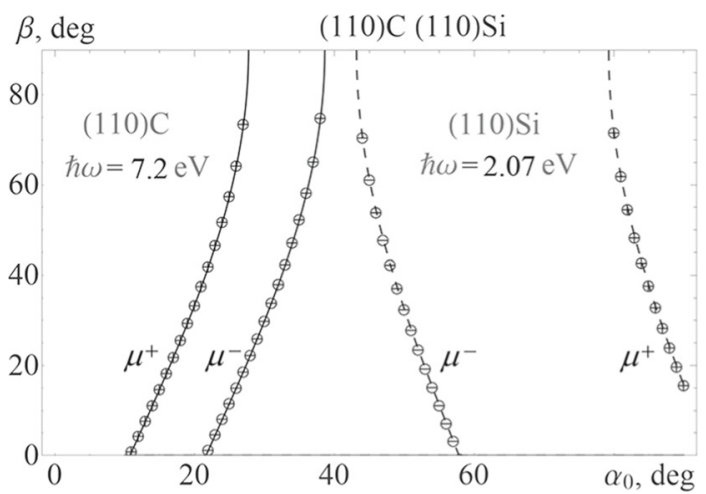

Fig. 10 The emission angle $\beta$ for ChCR photon of the energy $\hbar \omega$ versus the muons inlet angle $\alpha_{0}$ in a crystal

ChCR photons of the energy $\hbar \omega=7.2 \mathrm{eV}$ in a diamond $\mathrm{C}$ crystal as well as $\hbar \omega=2.07 \mathrm{eV}$ in a Si crystal. The graphs are drawn for the angles $\theta$, at which ChCR photons are emitted (see Tables 1 and 2).

The results obtained are mostly thickness dependent. In our analysis the crystals chosen are characterised by high transmission, hence, the thickness dependence is mainly defined by the projectiles scattering in various crystals at well defined directions of the motion. As we are studying the motion known as channeling, when the undulating projectile trajectory is determined by the continuous channeling potential, the scattering at which is thermodynamically described by "the dechanneling length" $[10,24]$. In other words, dechanneling length reflects the finite lifetime for a projectile in a given channeling state. Thus, the intensity of radiation emission by various particles under channeling regime depends not only on the gradient of scattering potential, which, for instance, for negative particles is typically higher, but also on the particle channeling lifetime, which, on the contrary, is typically longer for positive particles. Anyhow, the analysis should be performed in details for each case separately that becomes especially important for our task on positive and negative muons.
If to plan some experimental verification for newly described radiation, obviously, the choice of particles is primarily determined by experimental capabilities - doubtly there are such experimental groups that can provide any conditions that are required. From the point of view of the dechanneling length aforementioned, protons are most preferable - for heavier particles, the dechanneling length at equal energy is longer. However, potentially, electrons have the highest yield of ChCR. Therefore, probably, muons can be considered the golden middle to detect $\mathrm{ChCR}$ for the first time.

\section{References}

1. V.D. Shiltsev, Int. J. Mod. Phys. A 34(34), 1943002 (2019)

2. K.B. Korotchenko, Y.L. Pivovarov, Y. Takabayashi, S.B. Dabagov, Phys. Lett. B 795, 592 (2019)

3. B.M. Bolotovskii, Phys. Usp. 52, 1099 (2009)

4. G.N. Afanasiev, Vavilov-Cherenkov and Synchrotron Radiation (Springer Science \& Business Media, Berlin, 2004)

5. J.D. Jackson, Classical Electrodynamics, 3rd edn. (Wiley, New York, 1998)

6. G.F. Knoll, Radiation Detection and Measurement (Wiley, New York, 2010)

7. E. Graverini and On Behalf of the ATLAS, CMS and LHCb Collaborations, J. Phys. Conf. Ser. 1137, 012025 (2019)

8. V.V. Beloshitsky, M.A. Kumakhov, Sov. Phys. JETP 47(4), 652 (1978)

9. V.V. Beloshitsky, S.B. Dabagov, Sov. Phys. Tech. Phys. 33, 939 (1988)

10. S.B. Dabagov, N.K. Zhevago, La Rivista del Nuovo Cimento 31(9), 491 (2008)

11. K.B. Korotchenko, Y.L. Pivovarov, JETP Lett. 103(2), 8793 (2016)

12. K.B. Korotchenko, Y.L. Pivovarov, Phys. Lett. A 382, 444 (2018)

13. R.A. Carrigan Jr., Int. J. Mod. Phys. A 25(1), 55 (2010)

14. C. Ankenbrandt et al., Phys. Rev. STAB 2, 081001 (1999)

15. C. Ankenbrandt et al., Muon Collider Task Force Report FermilabTM-2399-APC (2007). https://doi.org/10.2172/923068

16. J. Beringer et al. (Particle Data Group), Phys. Rev. D 86, 010001 (2012)

17. C. Patrignani et al. (Particle Data Group), Chin. Phys. C 40, 100001 (2016)

18. M.N. Polyanskiy, Refractive index database. Available at https:// refractiveindex.info

19. Xiao Lin et al., Nat. Phys. 14, 816 (2018)

20. F.J. García de Abajo et al., Phys. Rev. Lett. 91, 143902 (2003)

21. G. Adamo et al., Phys. Rev. Lett. 103, 113901 (2009)

22. A.M. Cook et al., Phys. Rev. Lett. 103, 095003 (2009)

23. Fang Liu et al., Nat. Photonics 11, 289 (2017)

24. M.A. Kumakhov, Radiation from Charged Particles in Solids (AIP, New York, 1989) 